\title{
Transcriptomic analysis reveals the GRAS family genes respond to gibberellin in Salvia miltiorrhiza hairy roots
}

\author{
Wenrui Li ${ }^{1,2}$, Chuangfeng Liu ${ }^{3}$, Jingling $\mathrm{Liu}^{3}$, Zhenqing $\mathrm{Bai}^{3}$ and Zongsuo Liang ${ }^{1,4^{*}}$ (D)
}

\begin{abstract}
Background: Salvia miltiorrhiza is one of the most important traditional Chinese medicinal plants with high medicinal value. Gibberellins are growth-promoting phytohormones that regulate numerous growth and developmental processes in plants. However, their role on the secondary metabolism regulation has not been investigated.

Results: In this study, we found that gibberellic acid (GA) can promote hairy roots growth and increase the contents of tanshinones and phenolic acids. Transcriptomic sequencing revealed that many genes involved in the secondary metabolism pathway were the GA-responsive. After further analysis of GA signaling pathway genes, which their expression profiles have significantly changed, it was found that the GRAS transcription factor family had a significant response to GA. We identified 35 SmGRAS genes in S. miltiorrhiza, which can be divided into 10 subfamilies. Thereafter, members of the same subfamily showed similar conserved motifs and gene structures, suggesting possible conserved functions.
\end{abstract}

Conclusions: Most SmGRAS genes were significantly responsive to GA, indicating that they may play an important role in the GA signaling pathway, also participating in the GA regulation of root growth and secondary metabolism in S. miltiorrhiza.

Keywords: Transcriptome, GRAS family, Gibberellin, Salvia miltiorrhiza hairy roots, Secondary metabolism

\section{Background}

Salvia miltiorrhiza Bunge (Danshen) is a well-known traditional Chinese medicine with high medicinal and economic value. It is mainly used to treat cardiovascular and cerebrovascular diseases [1]. The Chinese pharmacopeia stipulates that the medicinal part of S. miltiorrhiza is its dried root. There are two major bioactive components of S. miltiorrhiza, lipophilic tanshinones and hydrophilic phenolic acids [2]. More than 40

\footnotetext{
*Correspondence: liangzs@ms.iswc.ac.cn

'Institute of Soil and Water Conservation, Chinese Academy of Sciences and Ministry of Water Resources, Yangling 712100, China

${ }^{4}$ College of Life Sciences and Medicine, The Key Laboratory of Plant

Secondary Metabolism and Regulation of Zhejiang Province, Zhejiang Sci-Tech University, Hangzhou 310018, China

Full list of author information is available at the end of the article
}

tanshinones and 20 hydrophilic phenolic acids have been isolated and identified from S. miltiorrhiza [3]. The tanshinones, including dihydrotanshinone I (DT-I), cryptotanshinone $(\mathrm{CT})$, tanshinone I (T-I) and tanshinone IIA (T-IIA), are biosynthesized via the mevalonic acid (MVA) and 2-C-methyl-D-erythritol-4-phosphate (MEP) pathways $[4,5]$. The phenolic acids, including salvianolic acid B (Sal B) and rosmarinic acid (RA), are biosynthesized through the phenylpropanoid and tyrosine-derived pathways [6, 7]. Most of the key biosynthetic enzyme genes of those pathways have been identified $[8,9]$. But the limited supply of bioactive compounds is not able to meet the ever-increasing market demand. Due to this, methods for improving the secondary metabolites biosynthesis have been tried, such as adversity stress, 
addition elicitor, overexpressing or suppressing genes codifying to enzymes or transcription factors involved in the biosynthetic pathways of these secondary metabolites. Nonetheless, the regulation of gibberellin on secondary metabolites biosynthesis remains unknown.

GAs are growth-promoting phytohormones that regulate numerous growth and developmental processes throughout the whole life cycle of plant, including seed germination, root and stem elongation, and flower development [10]. Since the 1950s, more than 130 GAs have been identified in various plants (http://www.plant-hormones.info/gibberellin_nomenclature.htm) [11]. However, only a few of them, such as G1, G3, G4 and G7, are bioactive [10]. GAs biosynthesis and catabolism pathways in plants have been well characterized. GAs are biosynthesized from the common precursor transgeranylgeranyl diphosphate (GGPP), formed via the MEP pathway. Then, GGPP is modified through the sequential action of two terpene cyclases CPS and KS, followed by oxidation by cytochrome P450 monooxygenases and 2-oxoglutarate-dependent dioxygenases, finally forming GA [12]. Subsequently, GA become functional on plants through its signaling pathways [13]. Binding of GA to the receptor, GID1, causes a conformational change in the $\mathrm{N}$-terminal of protein, which promotes its association with the GRAS domain of DELLA protein. This stable complex enables an efficient SCF ${ }^{\text {SLY1 }}$ recognition and subsequent degradation of DELLA by the proteasome [14].

The plant-specific GAI-RGA-SCR (GRAS) proteins family function as transcriptional regulators, playing key roles in the GA signaling. Most GRAS proteins contain an $\mathrm{N}$-terminal less-conserved variable region and a $\mathrm{C}$ terminal conserved GRAS domain. Typical GRAS domains comprise 5 conserved sequence motifs: leucine heptad repeats I (LHRI), VHIID, leucine heptad repeats II (LHRII), PFYRE and SAW [15]. Flanked by two leucine-rich regions, the VHIID motif is present in all GRAS family members. Based on their amino acid sequences, the GRAS family is divided into 10 distinct subfamilies: DELLA, SCL3, LAS, SCL4/7, SCR, SHR, SCL9 (LISCL), HAM, PAT1, and DLT [16]. Protein sequences in different subfamily have different characteristics and perform different functions. For example, DELLA proteins possess a conserved DELLA sequence motif in the N-terminal region, which function as GA repressors, acting as key regulatory targets in the GA signaling pathway during the growth regulation $[17,18]$. SCL3, in turn, functions as a repressor of DELLA, which can positively regulate the GA signaling pathway and control GA homeostasis during the Arabidopsis thaliana root development $[19,20]$. The SCL subfamily participates in root cell elongation, also in GA/DELLA signaling and on the stress response mechanisms [15]. The
VHIID and PFYRE motifs in the GRAS domain of SHR are essential for the interaction between SCR and SHR [16]. They could form a complex in order to participate in regulating root-related developmental processes in Arabidopsis [21, 22]. The PAT1 subfamily has been shown to mediate phytochrome and defense signaling pathways [23]. Moreover, LISCL has two conserved subfamily-restricted acidic motifs in the $\mathrm{N}$-domain and has been reported to be involved in stress response as well as in adventitious root formation in response to auxin [16].

Although GA plays an important role in many aspects of plant growth and development, little is known about its role in regulating secondary metabolism. As diterpenoids, GA and tanshinones have common precursor GGPP [9]. There might be some correlation between the metabolic processes of GA and tanshinones. In addition, GRAS has crucial roles in the GA signaling pathway. Our previous research has shown that 3 SmGRAS genes significantly promote tanshinones biosynthesis, also inhibiting GA biosynthesis in hairy roots of $S$. miltiorrhiza $[24,25]$. Therefore, we speculated that SmGRASs might mediate the regulation of secondary metabolism by the GA signaling in S. miltiorrhiza. In order to fully understand the role of the SmGRASs family genes on the regulation of secondary metabolism by GA signaling, the hairy roots of wild type $S$. miltiorrhiza were treated with GA, followed with determination of changes in root biomass, root diameter, the contents of tanshinones and phenolic acids. Meanwhile, we also measured the transcriptomic changes and specifically analyzed the transcriptional level alterations of the secondary metabolic pathway and GA signaling pathway genes. Finally, the bioinformatics of all SmGRAS family genes in S. miltiorrhiza and their responses to GA were analyzed. Our results revealed the possible pathways in which GA regulating secondary metabolism, as well as the response of $S m G R A S s$ to GA on this secondary metabolism regulation in $S$. miltiorrhiza, providing a reference for the GA signaling pathway to regulate secondary metabolism.

\section{Results}

\section{GA treatment affects root growth and secondary} metabolism

The S. miltiorrhiza hairy roots were treated with GA during their growth. After 6-day cultivation, the fresh and dry weights of the GA-treated hairy roots were all significantly higher than the controls (Fig. 1a-c), increasing by 19 and 13\%, respectively. Moreover, the root diameter of GA-treated hairy roots was significantly higher than that of the controls, increasing by $25 \%$ (Fig. 1d). These results indicated that the GA application promoted the growth of hairy roots. In order to investigate the changes of secondary metabolites affected by GA, 
A

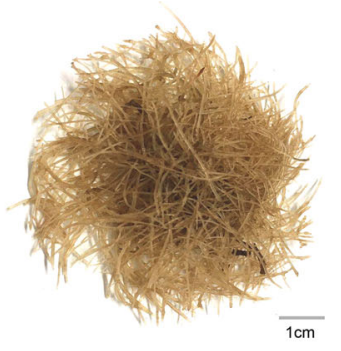

CK

D

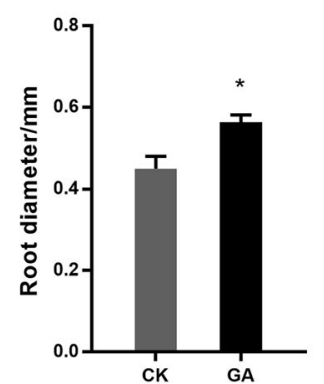

E

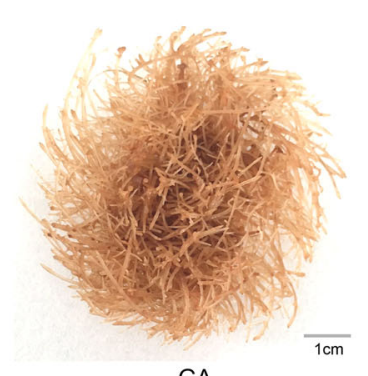

GA

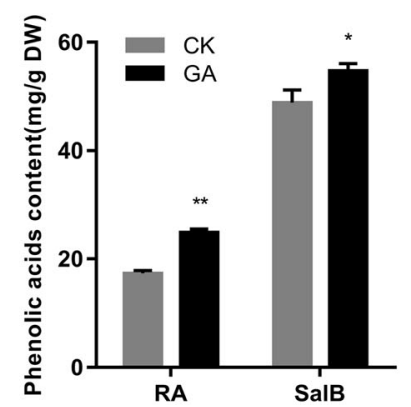

B

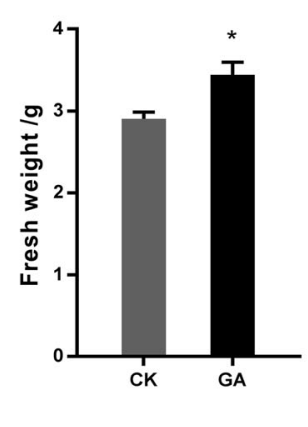

F

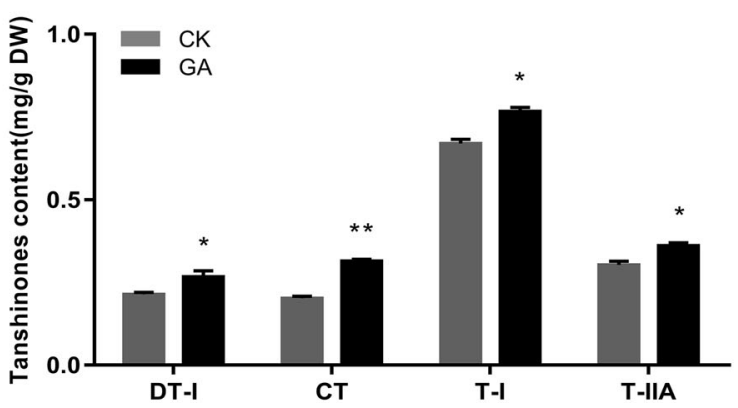

Fig. 1 Phenotype, root biomass, root diameter and secondary metabolites contents in S. miltiorrhiza hairy roots under CK and GA treatment. a Phenotype of hairy roots under CK and GA treatment for 6 days. b Fresh weight of hairy roots under CK and GA treatment for 6 days. c Dry weight of hairy roots under CK and GA treatment for 6 days. $\mathbf{d}$ Root diameter of hairy roots under CK and GA treatment for 6 days. e Phenolic acids content of hairy roots under CK and GA treatment for 6 days. $\mathbf{f}$ Tanshinones content of hairy roots under CK and GA treatment for 6 days

the contents of phenolic acids and tanshinones were determined after GA treatment. The contents of two phenolic acids and four tanshinones in the hairy roots were all significantly increased in the GA-treated hairy roots (Fig. 1e, f). The increase rates in RA and Sal B were 46 and $14 \%$, respectively, while the increase rates in four tanshinones were $25 \%$ (DT-I), 55\% (CT), 15\% (T-I) and $20 \%$ (T-IIA). Collectively, the data indicated that GA treatment promoted the root growth, also increased the accumulation of phenolic acids and tanshinones in the $S$. miltiorrhiza hairy roots.

\section{Transcriptome-scale analysis of GA-responsive genes}

In order to gain a comprehensive overview of the GAresponsive genes, we performed a transcriptomic analysis of CK and GA-treated hairy roots. 10321 differentially expressed genes (DEGs) were found and annotated in the volcano plot. The comparison of CK and GA-treated hairy roots revealed that 4945 genes were GA-induced, and 5376 were GA-repressed (Fig. 2a). To verify the results reliability from RNA-seq, 10 genes were randomly selected for Quantitative Reverse-Transcription PCR (qRT-PCR) analysis, whose results were consistent with the RNA-seq results, indicating that these RNA-seq data was reliable (Fig. S1, 2 and 3, Table S1, 2). The global functional analysis of the DEGs revealed that the "biological processes", "metabolic processes" and "cellular processes" were the top three categories in the most enriched gene ontology (GO) terms (Fig. 2b, Table S3). Additionally, these DEGs identified were further assessed using the Kyoto Encyclopedia of Genes and Genomes (KEGG). The most significantly enriched term was "biosynthesis of secondary metabolites", followed by "ribosome", "plant-pathogen interaction" and some "primary and secondary metabolic pathways" (Fig. 2c, Table S4).

\section{Secondary metabolism pathway genes in response to GA treatment}

In order to further explore the effect of GA on secondary metabolism, we used the Mapman program to analyze the transcriptomic data (Fig. 3). The results showed that most DEGs of secondary metabolism were GA-induced, especially the shikimate pathway, MVA pathway, simple phenols, betaines, wax, and anthocyanins. Diterpenoids, such as GA and tanshinones, are biosynthesized by MVA and MEP pathways. Most of the DEGs in the MVA pathway were GA-induced at the transcriptional level, which might be related to the fact that GA-induced tanshinones biosynthesis. On the other hand, phenolic acids are mainly biosynthesized by shikimate and phenylpropane pathways. The results showed that most of the shikimate pathway DEGs and some of the phenylpropane pathway DEGs were induced by GA. In addition, GA also affected the biosynthesis pathways 


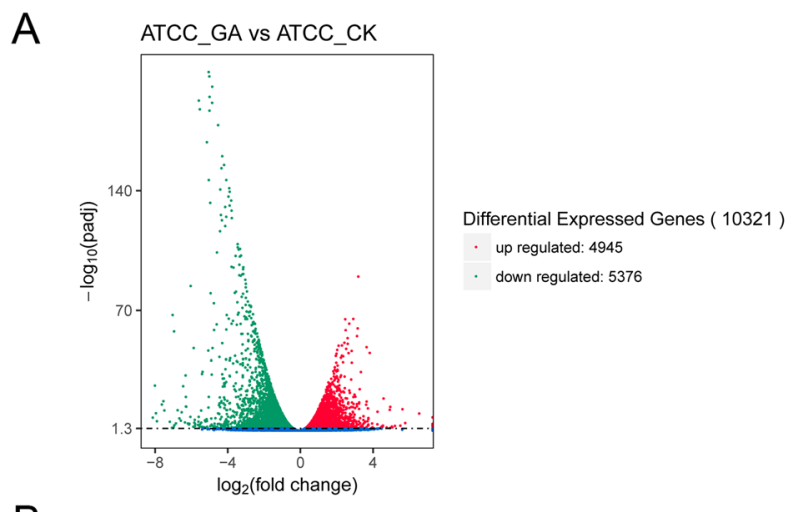

B

The Most Enriched GO Terms (ATCC_GAvsATCC_CK)

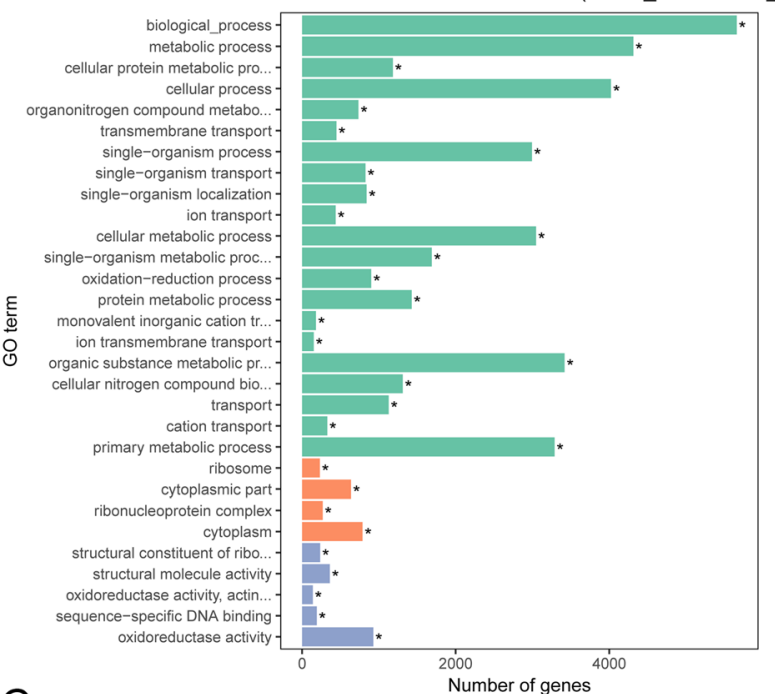

type

biological_process

cellular_component

molecular_function

C

Number of genes
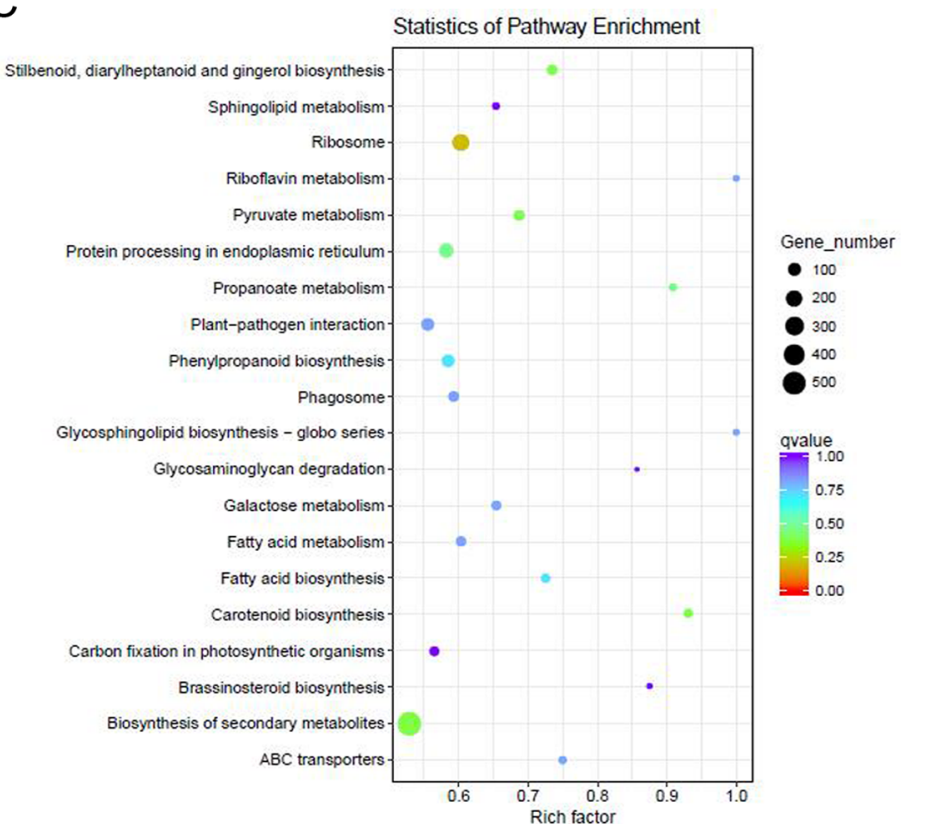

Fig. 2 (See legend on next page.) 
(See figure on previous page.)

Fig. 2 Transcriptomic profiling analysis under CK and GA treatment in hairy roots. a Volcano plots of the differentially expressed genes (DEGs) in the comparison of GA (ATCC-GA) and CK (ATCC-CK) hairy roots. $\mathbf{b}$ Functional gene ontology (GO) term classifications of DEGs from comparisons of GA and CK hairy roots. c Kyoto Encyclopedia of Genes and Genomes (KEGG) classification of DEGs in the comparisons of GA and CK hairy roots

of flavonoids and alkaloids-like, wax and glucosinolates. In summary, GA regulated the gene transcription in many secondary metabolites' biosynthetic pathway.

\section{GA biosynthetic and signaling pathways genes in response to $\mathrm{GA}$ treatment}

GA treatment can directly affect the regulation of the GA signaling pathway in plant. To investigate the effect of this treatment on GA biosynthesis and signaling pathway, we further analyzed and summarized the DEGs involved in GA biosynthetic and signaling pathway (Fig. 4). The results showed that the transcriptional changes in GA biosynthetic pathway genes were diverse, some DEGs had increased their expression levels, whereas others had it decreased. In the downstream GA signaling pathway, the expression of GID1 (GA receptor) was increased. Coherently, the expression levels of most GRAS family genes, which were the key regulators of the GA signaling pathway, were increased as well. Nevertheless, the expressions of F-box proteins SCF in the GA signaling pathway were different under GA treatment. In conclusion, GA could effectively regulate the expressions of biosynthetic pathway genes and downstream signaling pathway genes, especially the SmGRAS family genes, and further regulating many downstream physiological processes, such as cell growth, secondary metabolism, and plant resistance.

\section{Identification and phylogenetic analysis of GRAS proteins} in S. miltiorrhiza

In order to study the roles of SmGRAS in GA regulation of root growth and secondary metabolism of S. miltiorrhiza, we conducted a comprehensive analysis of the SmGRAS family genes in this species. We used HMMER to screen the protein sequences based on the HMM profiles from the $S$. miltiorrhiza genome database to identify putative GRAS proteins. Thirty-five SmGRAS proteins were identified from it, which were named GRAS1 $~ 35$. The putative amino acid sequences of SmGRAS1 35 contained the conserved GRAS domain for the GRAS protein [15]. To study the evolutionary relationships of SmGRAS genes, phylogenetic tree analysis with dicotyledons of Arabidopsis and monocotyledons of rice was constructed, which revealed that the SmGRAS proteins were divided into 10 subfamilies (Fig. 5). Among these GRAS family proteins, there were 9 proteins from the PAT1 subfamily, 6 proteins from the LISCL subfamily, 5 proteins from the SHR subfamily, 4 proteins from the SCL and DELLA subfamilies, 2 proteins from the DLT and SCR subfamilies, bedides the other 3 subfamilies

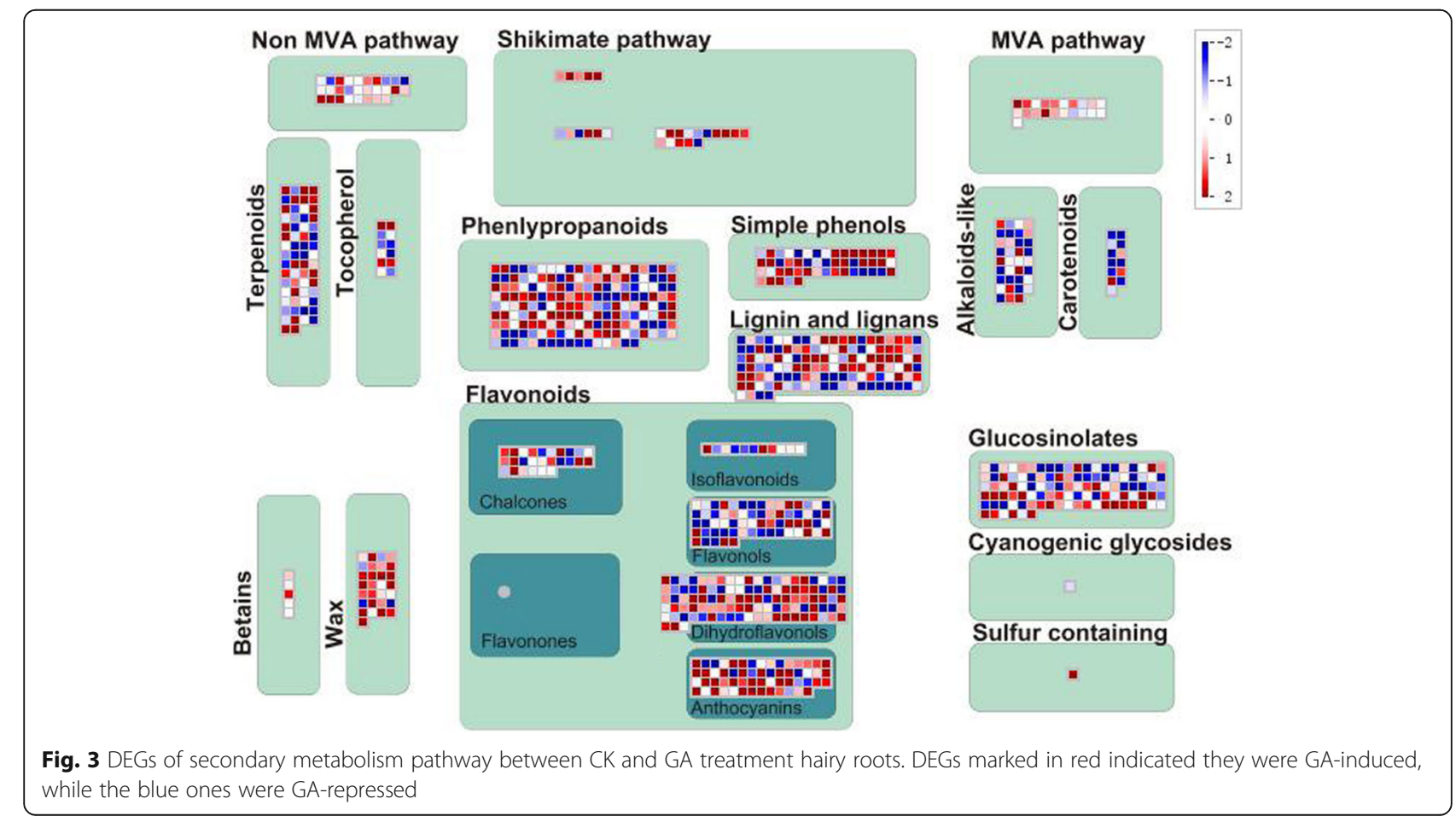




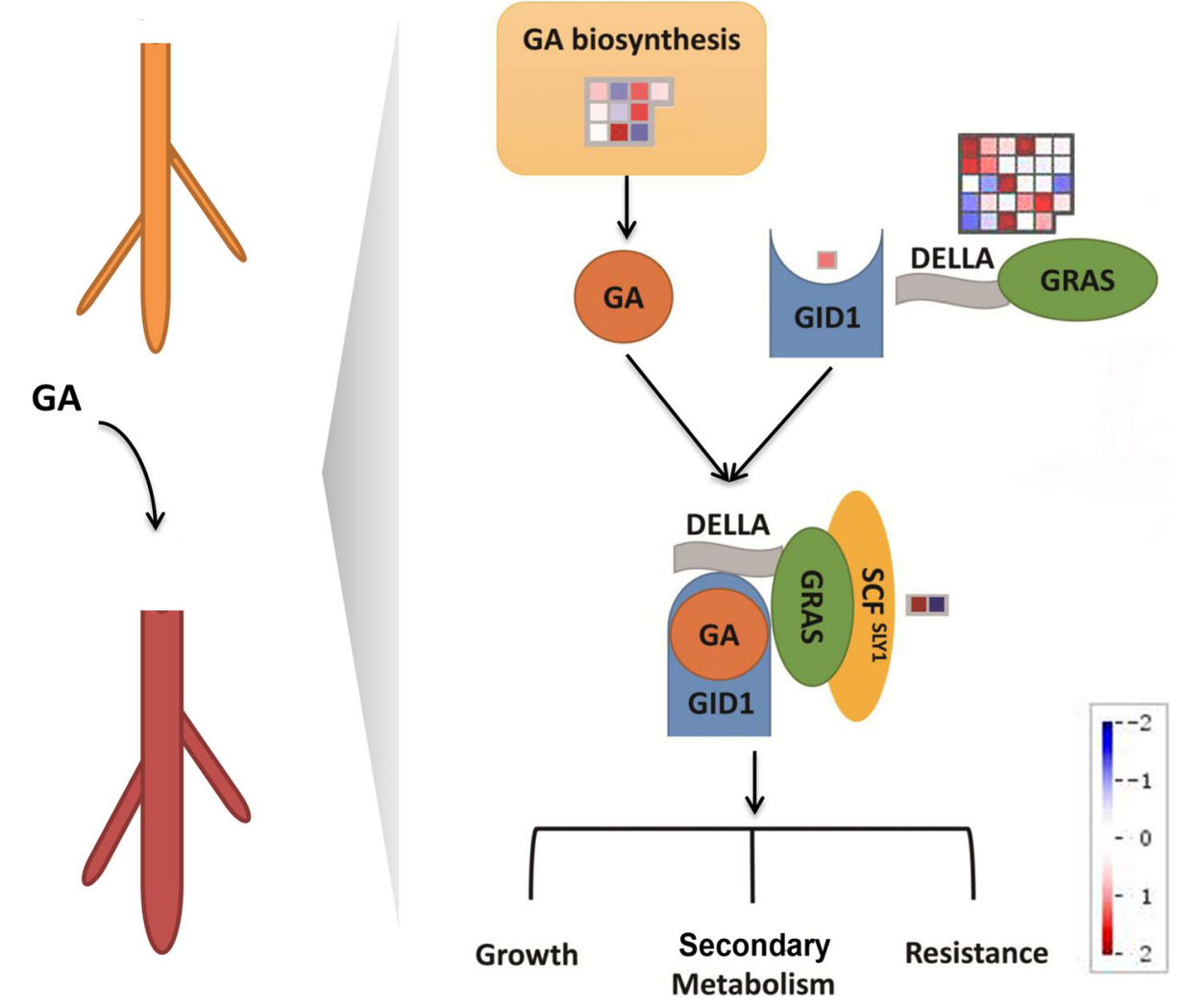

Fig. 4 A model for a possible mechanism of the regulation of GA to root growth and secondary metabolism. DEGs marked in red indicated they were GA-induced, while the blue ones were GA-repressed

only having 1 protein. Therefore, we speculated that these SmGRAS genes might be similar to Arabidopsis GRAS genes of the same subfamily. They might be involved in the GA signaling pathway, root and stem cortex development, light morphogenesis and stress tolerance.

\section{SmGRAS proteins sequence alignments and conserved motifs}

In order to further confirm that these 35 genes belong to the GRAS family, we used DNAMAN and online MEME to perform multiple sequences alignment and conservative domain analysis on them. The multiple sequences alignment result showed that the amino acid sequences of all these 35 proteins have high identities (Fig. 6a). Almost all those SmGRAS proteins contain conserved GRAS domain, as LHRI, VHIID, LHRII, PFYRE and SAW. We also used online MEME to identify the conserved motifs of full-length SmGRAS proteins (Fig. 6b, c) and 10 most conserved motifs located in the GRAS domain were shown in Fig. 6b. In Fig. 6c, there are 20 conserved motifs were identified in the 35 SmGRAS proteins. The VHIID and SAW motifs which were the most conserved motifs in the GRAS domain, were found in all SmGRAS proteins. The LHRI, LHRII, and PFYRE motifs were found in most of SmGRAS proteins, while other 15 motifs were found just in some SmGRAS proteins. The results suggested that all these 35 SmGRAS proteins have the conserved GRAS domain, however, SmGRAS proteins from different subfamilies have different motifs, probably in order to perform different functions.

\section{Structural analysis of SmGRAS genes}

To further analyze the structural components and physicochemical properties of $35 S m G R A S$ genes, we conducted an in-depth analysis with ExPASy (Table 1). The result showed that the open reading frame length of most SmGRAS family genes was over $1000 \mathrm{bp}$. About the $35 \operatorname{SmGRAS}$ genes, SmGRAS34 was the shortest one, with $453 \mathrm{bp}$, while the longest one was the SmGRAS3, with $2247 \mathrm{bp}$. The protein length ranged from 150 (SmGRAS34) to 748 amino acids (SmGRAS3), and the molecular weight ranged from 16.8 to $84.0 \mathrm{kDa}$. The protein isoelectric points ranged from 4.8 (SmGRAS20) to 9.0 (SmGRAS35), with most of them ranging from 5 to 6 . The majority of $S m G R A S$ proteins contained just 1 exon, while some contained 2 exons. Only the SmGRAS28 has 4 exons and SmGRAS35 has 5 exons. 


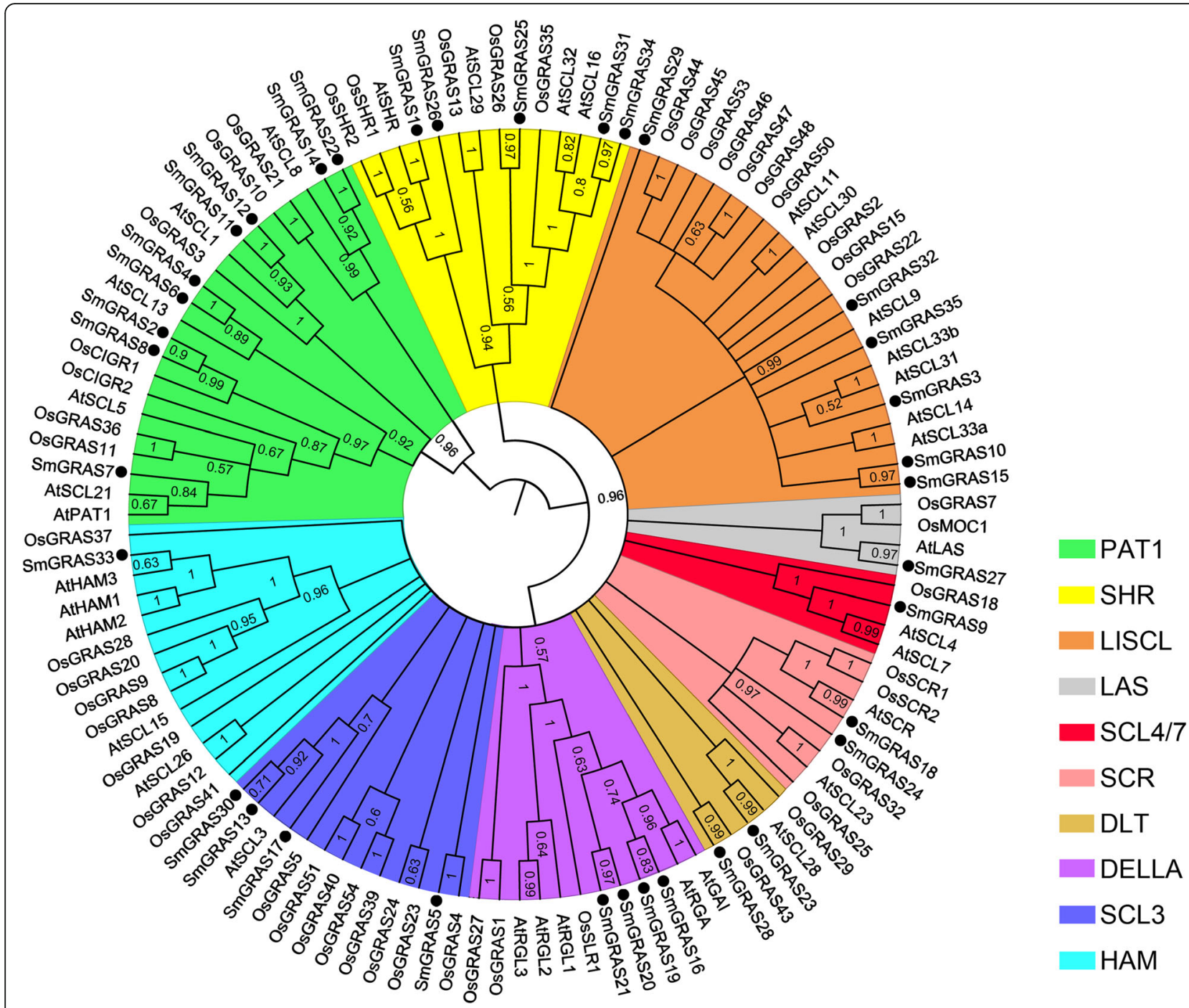

Fig. 5 The phylogenic tree of GRAS transcription factors used the Neighbor-Joining (NJ) method of S. miltiorrhiza, Arabidopsis, and rice. Different subfamilies were marked with different background colors

\section{Expression analysis of SmGRAS genes in response to GA treatment}

As the key regulator of the GA signaling pathway, the transcription levels of GRASs were significantly affected by GA. We comprehensively analyzed the transcription level changes of these $35 \operatorname{SmGRAS}$ family genes after $100 \mu \mathrm{m}$ GA treatment for $2 \mathrm{~h}$. So these expression levels changed a lot. The heatmap showed that 15 SmGRAS genes were GA-induced, while 11 SmGRAS genes were GA-repressed, besides other 9 genes did not change significantly under GA treatment (Fig. 7). The most significantly increased of these was SmGRAS5, which increased 4-fold expression levels, followed by SmGRAS20 (2.3-fold) and SmGRAS14 (2.1-fold). At the other hand, the most significantly reduced in those genes was the SmGRAS28, which reduced about $90 \%$ in comparison with the control. The expressions of
SmGRAS31, SmGRAS8, SmGRAS11, and SmGRAS12 were all fell by more than half.

\section{Discussion}

It is well known that bioactive GAs are diterpene phytohormones that regulate plant growth and development throughout the whole life cycle [14]. There are many reports concerning the important roles of GA in plant growth, development and stress tolerance [10-13], but just few about the relationship between GA and secondary metabolism. In addition, GA shares the identical biosynthetic pathway and precursor substances with diterpenoid metabolites tanshinones, which were the main secondary metabolites of S. miltiorrhiza [9]. We speculated that there might be some correlation between GA and tanshinones. Therefore, we treated the hairy roots of $S$. miltiorrhiza with GA and found that it not 


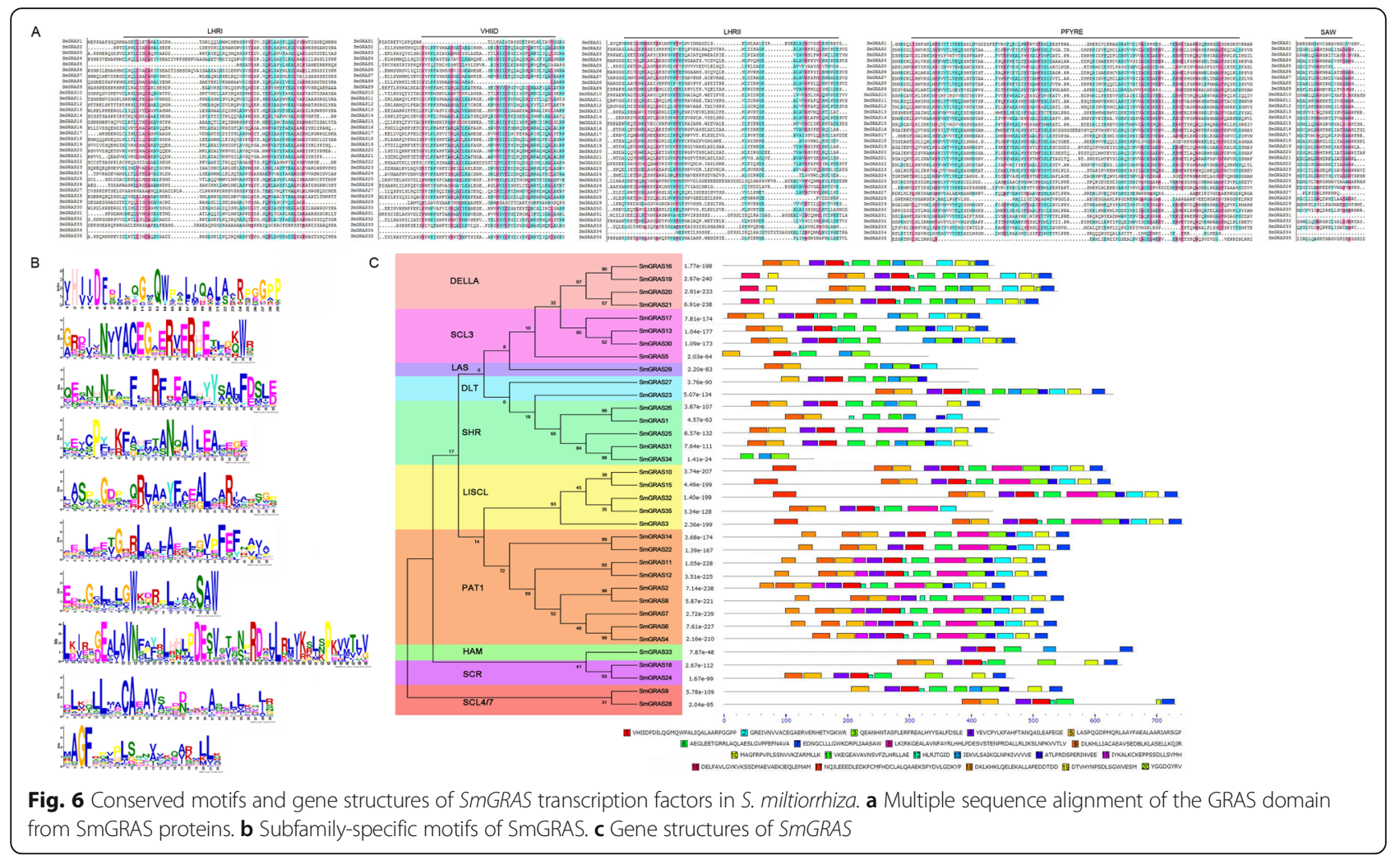

only promoted the root growth but also increased the accumulation of tanshinones and phenolic acids. These results make us beginning to pay attention to the specific mechanism of GA regulating secondary metabolism.

It is also known that GA regulates many growth and development processes such as seed germination, root and shoot elongation, metabolism, stress tolerance, flowering and fruit patterning $[10,12]$. The GA signaling pathway includes the biosynthesis of the active GA, perception, signal transduction and inactivation [12]. It is now clear that GAs accumulate in the elongating endodermal cells of Arabidopsis root, playing central roles in growth regulation through the key transcription factor DELLA (GRAS family member) [10, 13]. Several genes of the GRAS transcription factor family in the GA signaling pathway have been reported to regulate the development of the cortex in the root. For instance, GRAS family genes form a SHR-SCR-SCL3 complex, which regulates the middle cortex formation in Arabidopsis root [26]. SCL28 also plays an important role in root growth response to stress-induced microtubule organization in Arabidopsis [27]. In addition, the GAmediated growth control and energy metabolism have an interaction, which coordinates cell wall extension, secondary metabolism and lipid metabolism in Arabidopsis [28]. Similarly, the overexpression of HaGRASL reduces the GAs metabolic flow in Arabidopsis, so that modification could be relevant in axillary meristem development [29].

Based on this reasoning, after treating wild-type hairy roots of S. miltiorrhiza with GA, we found that it promoted the root growth, enhanced the accumulation of tanshinones and phenolic acids. In order to explore the possible molecular mechanism, we performed transcriptomic analysis, whose results showed that the gene response of the secondary metabolic pathway was significant after GA treatment. So we speculated that GA regulated the accumulation of secondary metabolites by regulating the expression of genes involved in the secondary metabolites biosynthetic pathway. After further analysis of the DEGs in secondary metabolic pathways, we found that most DEGs in MVA, shikimate and phenylpropane pathways are GA-induced at the transcriptional level. This result also explains a series of composition content changes in the hairy root of S. miltiorrhiza at the transcriptional level after GA treatment, such as the increased tanshinones and phenolic acids contents. In addition, since our previous research found that 3 SmGRAS genes can promote the biosynthesis of tanshinones and inhibit the biosynthesis of GA, we hypothesize that GRAS core regulators of the GA signaling pathway would be involved in the regulation of GA on the secondary metabolism $[24,25]$. Therefore, we also deeply analyzed the expressions of DEGs in the GA signaling pathway. The results showed that the gene 
Table 1 The information of 35 SmGRAS transcription factors identified in S.miltiorrhiza genome

\begin{tabular}{|c|c|c|c|c|c|c|}
\hline Gene & Gene ID & ORF length (bp) & Protein length (aa) & Mw (Da) & $\mathrm{pl}$ & Exon no. \\
\hline SmGRAS1 & SMil_00008598 & 1470 & 489 & 54680.21 & 5.813 & 1 \\
\hline SmGRAS2 & SMil_00025700 & 1380 & 459 & 51215.14 & 6.184 & 1 \\
\hline SmGRAS3 & SMil_00006393 & 2247 & 748 & 83952.38 & 4.953 & 1 \\
\hline SmGRAS4 & SMil_00023920 & 1581 & 526 & 58112.72 & 5.427 & 1 \\
\hline SmGRAS5 & SMil_00019115 & 1041 & 335 & 37542.27 & 5.774 & 1 \\
\hline SmGRAS6 & SMil_00022108 & 1626 & 541 & 59758.38 & 5.462 & 1 \\
\hline SmGRAS7 & SMil_00020530 & 1563 & 520 & 57762.17 & 6.031 & 1 \\
\hline SmGRAS8 & SMil_00027641 & 1662 & 553 & 62006.62 & 5.661 & 2 \\
\hline SmGRAS9 & SMil_00022663 & 1653 & 550 & 60292.19 & 5.429 & 2 \\
\hline SmGRAS10 & SMil_00013932 & 1872 & 623 & 70517.82 & 6.435 & 1 \\
\hline SmGRAS11 & SMil_00010266 & 1575 & 524 & 58338.81 & 5.321 & 1 \\
\hline SmGRAS12 & SMil_00005963 & 1584 & 527 & 58587.25 & 5.121 & 1 \\
\hline SmGRAS13 & SMil_00014572 & 1308 & 435 & 48756.82 & 6.053 & 1 \\
\hline SmGRAS14 & SMil_00014379 & 1692 & 563 & 61920.17 & 6.916 & 1 \\
\hline SmGRAS15 & SMil_00013931 & 1905 & 634 & 71762.76 & 6.203 & 1 \\
\hline SmGRAS16 & SMil_00019995 & 1326 & 441 & 48523.73 & 5.853 & 1 \\
\hline SmGRAS17 & SMil_00012335 & 1269 & 422 & 47557.06 & 6.496 & 1 \\
\hline SmGRAS18 & SMil_00023609 & 1944 & 647 & 70502.62 & 5.986 & 2 \\
\hline SmGRAS19 & SMil_00027306 & 1617 & 538 & 59384.69 & 5.694 & 1 \\
\hline SmGRAS20 & SMil_00025750 & 1628 & 545 & 59449.16 & 4.832 & 1 \\
\hline SmGRAS21 & SMil_00015621 & 1548 & 515 & 56501.40 & 5.806 & 1 \\
\hline SmGRAS22 & SMil_00016132 & 1695 & 564 & 61825.58 & 5.768 & 2 \\
\hline SmGRAS23 & SMil_00019107 & 1911 & 636 & 70786.90 & 6.863 & 1 \\
\hline SmGRAS24 & SMil_00011905 & 1419 & 472 & 52047.14 & 6.533 & 1 \\
\hline SmGRAS25 & SMil_00024909 & 1326 & 441 & 48314.18 & 5.097 & 1 \\
\hline SmGRAS26 & SMil_00017304 & 1266 & 421 & 47024.52 & 5.397 & 1 \\
\hline SmGRAS27 & SMil_00018880 & 1206 & 401 & 44337.81 & 8.832 & 2 \\
\hline SmGRAS28 & SMil_00018052 & 2205 & 734 & 81680.17 & 6.038 & 4 \\
\hline SmGRAS29 & SMil_00012105 & 1248 & 415 & 44237.29 & 9.695 & 2 \\
\hline SmGRAS30 & SMil_00000751 & 1440 & 479 & 53599.74 & 6.116 & 1 \\
\hline SmGRAS31 & SMil_00012472 & 1218 & 405 & 44533.13 & 5.761 & 1 \\
\hline SmGRAS32 & SMil_00017280 & 2235 & 744 & 83995.86 & 5.793 & 1 \\
\hline SmGRAS33 & SMil_00000430 & 1998 & 665 & 71197.52 & 5.928 & 2 \\
\hline SmGRAS34 & SMil_00006319 & 453 & 150 & 16797.00 & 4.990 & 1 \\
\hline SmGRAS35 & SMil_00004080 & 1326 & 441 & 49830.65 & 9.001 & 5 \\
\hline
\end{tabular}

expression levels of most GRAS family genes and GA receptor GID1 in the pathway were significantly increased. Therefore, it is speculated that GA induction affects the GA signaling pathway, also causing the response of SmGRASs in the GA signaling pathway, regulating some downstream physiological processes such as growth, secondary metabolism and stress response.

In order to study the roles of GRAS during the GA regulation on plant growth and secondary metabolism, we used HMMER to search for all SmGRAS protein sequences based on the HMM profiles from the S. miltiorrhiza genome database. SmGRAS was found to be a large family of transcription factors with 35 genes. These SmGRAS proteins were systematically analyzed in relation to the GRAS family proteins of monocotyledons rice and dicotyledons Arabidopsis. The phylogenetic tree revealed that these SmGRASs had gathered into 10 subfamilies, PAT1 (9 SmGRASs), LISCL (6), SHR (5), 


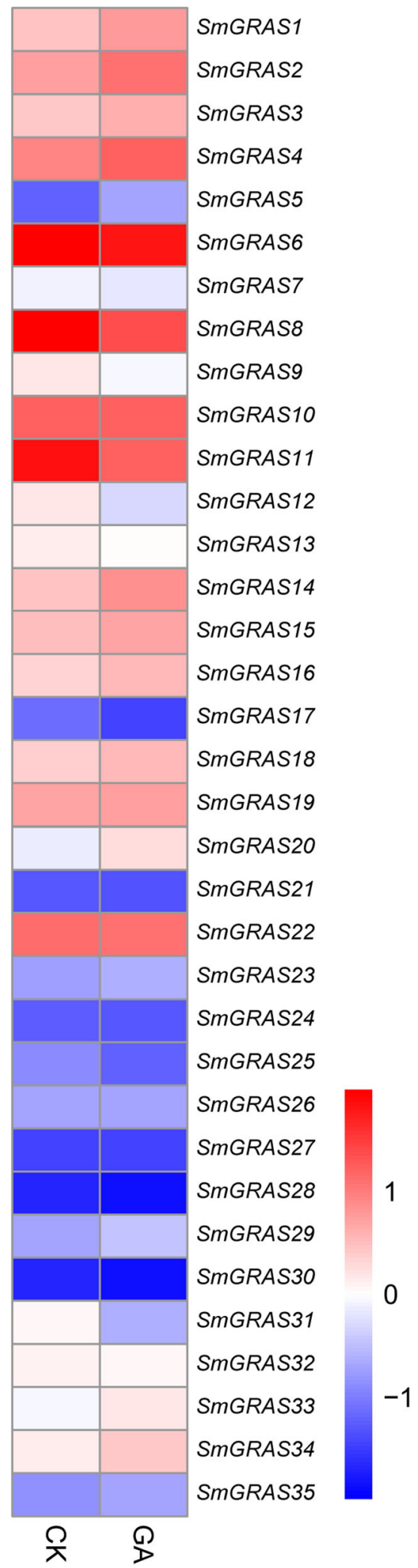

Fig. 7 Gene expression profiles of SMGRAS members under control and GA treatment. Genes marked in red indicated they were GAinduced, while the blue ones were GA-repressed
DELLA (4), SCL3 (4), SCR (2), DLT (2), HAM (1), SCL4/7 (1) and LAS (1). Orthologous genes generally retained similar functions. The PAT1 subfamily has been reported to mediate phytochrome, light and defense signaling pathways [15]. The LISCL subfamily was involved in stress response and response to auxin. SHR, SCR and SCL3 formed a complex to regulate middle cortex formation. DELLA has a central role in suppressing GA signaling [30]. DLT was involved in the brassinosteroid signaling, SCL4/7 was involved in the response to environmental stresses and LAS participated in the formation of lateral shoots [15]. Therefore, we speculated that SmGRASs might also be involved in the regulation of the above processes in S. miltiorrhiza. In addition, we further analyzed the conservative sequence. Most SmGRAS proteins are clustered into similar subfamilies, they share similar motifs, and also have family-specific functions. The conservative sequence is slightly different in different subfamilies. For example, motif 15 was only present in subfamily PAT1; motifs 10 and 16 were only present in DELLA subfamily; motif 17 was only found in subfamily LISCI, indicating their specific functions. The distribution of conserved motifs reflects the relations between different subfamilies. For example, motifs 13 and 20 were found, while motifs 8,15 and 17 were completely lost in subfamilies SCL3 and DELLA. These results indicated the evolutionary relationship between subfamilies SCL3 and DELLA is very close. Therefore, the structural analysis also provided a clue to determine the sub-family of GRAS genes and its ancient origin.

Finally, we analyzed the responses of all SmGRAS family genes to GA induction. The expression analysis indicated that most SmGRAS genes were significantly induced or inhibited in GA induction. In Arabidopsis, DELLA proteins act as repressors of GA-responsive plant growth and three DELLA genes (SmGRAS16, 19 and 20) were induced by GA treatment. SCL3, as the repressor of DELLA, could positively regulate the GA signaling pathway and also control GA homeostasis in Arabidopsis root [20]. SmGRAS13, 17 and 30 are orthologous to SCL3 gene in S. miltiorrhiza, so that it was evidently downregulated under GA treatment. Therefore, we speculated that SmGRAS proteins of different subfamilies played different regulatory roles through the GA signaling pathway, regulating many physiological processes in S. miltiorrhiza. Finally, we also proposed a model diagram for the GA signaling pathway regulation. The results showed that GA could regulate the expressions of biosynthetic pathway genes as well as the downstream signaling pathway genes, mainly SmGRAS transcription factors, and further regulating many downstream physiological processes, such as cell growth, secondary metabolism, and plant resistance. 


\section{Conclusions}

We found that GA could promote the growth of S. miltiorrhiza hairy roots, also increasing the accumulation of tanshinones and phenolic acids. Many DEGs in metabolic processes, especially the secondary metabolic processes, as well as the GA signaling pathway regulator SmGRASs could significantly be induced under GA treatment. The key regulatory factors SmGRASs play important roles on the GA-regulated root growth and secondary metabolism in S. miltiorrhiza. Although providing a range of references for understanding the molecular mechanism that exists behind the GA regulating secondary metabolism, further studies are needed for a better acquaintance of this complex mechanism.

\section{Methods}

\section{Plant materials and treatment}

The S. miltiorrhiza leaves were took from the sterile seedling line preserved at our laboratory at Northwest A\&F University in Yangling, Shaanxi Province, China. The establishment of $S$. miltiorrhiza hairy roots was derived from aseptic leaves of S. miltiorrhiza infected with Agrobacterium rhizogenes (ATCC15834), as previously reported [31]. Samples of the hairy roots ( $0.3 \mathrm{~g}$ fresh weight) were cultured in $100 \mathrm{~mL}$ beaker flasks contains $50 \mathrm{~mL}$ of the $6,7-\mathrm{V}$ liquid medium on an orbital shaker $120 \mathrm{rpm} \cdot \mathrm{min}^{-1}, 25^{\circ} \mathrm{C}$ in the dark, sub-cultured every 30 days.

For GA treatment, GA $_{3}$ (Sigma, CA, USA) stock solution was filter sterilized through $0.22 \mu \mathrm{m}$ filters and added to cultures of 21-day-old hairy roots, to a desired final concentration of $100 \mu \mathrm{M}$. After $2 \mathrm{~h}$ and 6 days of GA treatment, hairy roots were collected for the qRTPCR and HPLC analysis. Hairy roots without GA treatment were used as control samples. All treatments were performed in three independent biological replicates.

\section{HPLC analysis}

The 21-day-old hairy roots were treated with $100 \mu \mathrm{M}$ $\mathrm{GA}_{3}$ for 6 days and collected in three biological replicates. Then $0.04 \mathrm{~g}$ of dried hairy roots was extracted and analyzed by HPLC, according to the general method in our laboratory, which was previously described [32]. The $\mathrm{GA}_{3}$ concentration analysis was measured by HPLC as previously described as well [33].

\section{RNA-seq library construction and sequencing}

On $2 \mathrm{~h}$ after GA treatment, the GA-treated hairy roots (GA) and control samples (CK) were collected from three biological replicates and analyzed by transcriptomic-based technology. Total RNA was extracted using the RNA extraction kit following the manufacturer's instruction (Tiangen Biotech, Beijing, China). After measuring the quality, strand-specific RNA-Seq libraries were constructedand sequenced on the Illumina PE150 platform (Novogene,
Tianjin, China). The high-quality clean data were calculated and used for downstream analysis.

\section{Identification of DEGs and functional enrichment}

The reference genome and gene model annotation files of S. miltiorrhiza were downloaded from genome websites [34]. Fragments per kilobase of transcript per million mapped reads (FPKM) were used to determine the relative expression levels of each gene. DEGs were determined using the DESeq $R$ package [35]. The expression levels of DEGs were considered significantly differentially expressed genes with an adjusted $P$-value $<0.05$ and |foldchange $\mid \geq 2$. The GO term enrichment of DEGs was evaluated using the GOseq $\mathrm{R}$ package [36]. Statistical enrichment of DEGs in the KEGG was identified with KOBAS [37]. Then Mapman program was used to analyze the transcriptome data of metabolic and signal pathways.

\section{qRT-PCR validation}

A total of 10 DEGs were randomly chosen to verify the RNA-seq data (Fig. S3). Ten DEGs specific primers were designed by primer 5 (Table S5). qRT-PCR was performed on a real-time PCR system (Bio-Rad CFX96, USA), according to our previous methods [24]. The SmActin gene was used as the endogenous control [38]. All assays for each gene were performed in triplicate under identical conditions.

\section{Identification of GRAS family members in S. miltiorrhiza and phylogenetic analysis}

A Hidden Markov Model (HMM) of the GRAS domain (PF03514) was downloaded from the Pfam database (http://pfam.xfam.org). HMM algorithm (HMMER) was used (http://www.hmmer.org/) to search for the GRAS domain in the $S$. miltiorrhiza genome database [39], with an $E$-value $<1 \mathrm{e}^{-6}$. Multiple sequence alignments of GRAS protein sequences from $S$. miltiorrhiza, O. sativa and $A$. thaliana (https://phytozome.jgi.doe.gov/pz/portal.html) were performed using the Clustal X program. A phylogenetic tree based on the alignment was constructed with MEGA 6.0 by the neighbor-joining method with the bootstrap test ( $n=500$ replications) [40].

\section{Analysis of conserved motifs and gene structures}

Gene Structure Display Server (http://gsds.cbi.pku.edu. $\mathrm{cn} /$ ) was used to obtain the gene structure of introns and exons based on the CDS and corresponding to genic sequences. GRAS domains were aligned and the conserved sites were checked manually for their corresponding amino acid residues, which were shaded using DNAMAN software. Conserved motif analysis of SmGRASs was performed by online MEME tools with 20 motif numbers. The theoretical isoelectric point (pI) and molecular weight $(\mathrm{Mw})$ were predicted by the ExPASy server (http://web.expasy.org/computepi/). 


\section{Supplementary information}

Supplementary information accompanies this paper at https://doi.org/10. 1186/s12864-020-07119-3.

Additional file 1: Figure S1. The classification of raw reads. Additional file 2: Figure S2. The pearson correlation between sample replicates.

Additional file 3: Figure S3. Validating the different expression levels of the identified genes from RNA-seq data by qRT-PCR.

Additional file 4: Table S1. Data output quality list.

Additional file 5: Table S2. Reads and reference genome comparison list.

Additional file 6: Table S3. GO enrichment of DEGs between CK and GA-treated hairy roots. (XLS $3958 \mathrm{~kb}$ )

Additional file 7: Table S4. KEGG Pathwayenrichment of DEGs between CK and GA-treated hairy roots. (XLS 246 kb)

Additional file 8: Table S5. Primers used for qRT-PCR.

\section{Abbreviations}

GRAS: GAI-RGA-SCR; GA: Gibberellic acid; DT-I: Dihydrotanshinone I: CT: Cryptotanshinone; T-I: Tanshinone I; T-IIA: Tanshinone IIA; MVA: Mevalonic acid; MEP: 2-C-methyl-D-erythritol-4-phosphate; SAs: Salvianolic acids; Sal B: Salvianolic acid B; RA: Rosmarinic acid; GGPP: Trans-geranylgeranyl diphosphate; LHR: Leucine heptad repeats; DEGs: Differentially expressed genes; qRT-PCR: Quantitative Reverse-Transcription PCR; GO: Gene ontology; KEGG: Kyoto Encyclopedia of Genes and Genomes; FPKM: Fragments per kilobase of transcript per million mapped reads; HMM: Hidden Markov Model

\section{Acknowledgments}

We thank Federal University of Lavras, Rubens Diogo Junior for his valuable suggestions for revising the manuscript.

\section{Authors' contributions}

$W L$ and $Z L$ conceived and designed the experiment; $W L, C L, J$, and $Z B$ performed the experiments; WL analyzed the data and wrote the article with input from other authors. All authors read and approved the final manuscript.

\section{Funding}

This work was supported by the National Natural Science Foundation of China (No.81773835) for the design and analysis of the data in the study.

\section{Availability of data and materials}

The data sets supporting the conclusions of this article are included within the article and its additional files. Raw reads data has been deposited in NCBI under accession number PRJNA663993.

\section{Ethics approval and consent to participate}

Not applicable.

\section{Consent for publication}

Not applicable.

\section{Competing interests}

The authors declare that they have no competing interests.

\section{Author details}

${ }^{1}$ Institute of Soil and Water Conservation, Chinese Academy of Sciences and Ministry of Water Resources, Yangling 712100, China. ${ }^{2}$ School of Food and Biological Engineering, Shaanxi University of Science and Technology, Xi'an 710021, China. ${ }^{3}$ College of Life Sciences, Northwest A\&F University, Yangling 712100, China. ${ }^{4}$ College of Life Sciences and Medicine, The Key Laboratory of Plant Secondary Metabolism and Regulation of Zhejiang Province, Zhejiang Sci-Tech University, Hangzhou 310018, China.
Received: 15 January 2020 Accepted: 2 October 2020

Published online: 27 October 2020

\section{References}

1. Dong Y, Morrisnatschke SL, Lee KH. Biosynthesis, total syntheses, and antitumor activity of tanshinones and their analogs as potential therapeutic agents. Nat Prod Rep. 2011;28(3):529-42.

2. Xu J, Wei K, Zhang G, Lei L, Yang D, Wang W, Han Q, Xia Y, Bi Y, Yang M, Li M. Ethnopharmacology, phytochemistry, and pharmacology of Chinese Salvia species: a review. J Ethnopharmacol. 2018;225:18-30.

3. Zhang $Y$, Ji A, Xu Z, Luo H, Song J. The AP2/ERF transcription factor SmERF128 positively regulates diterpenoid biosynthesis in Salvia miltiorrhiza. Plant Mol Biol. 2019;100(1):83-93.

4. Guo J, Ma X, Cai Y, Ma Y, Zhan Z, Zhou Y, Liu W, Guan M, Yang J, Cui G, Kang L, Yang L, Shen Y, Tang J, Lin H, Ma X, Jin B, Liu Z, Peters RJ, Zhao Z, Huang L. Cytochrome P450 promiscuity leads to a bifurcating biosynthetic pathway for tanshinones. New Phytol. 2016;210(2):525-34.

5. Pei T, Ma P, Ding K, Liu S, Jia Y, Ru M, Dong J, Liang Z. SmJAZ8 acts as a core repressor regulating JA-induced biosynthesis of salvianolic acids and tanshinones in Salvia miltiorrhiza hairy roots. J Exp Bot. 2018;69(7):1663-78.

6. Petersen M. Rosmarinic acid: new aspects. Phytochem Rev. 2013;12(1):207-27.

7. Sun M, Shi M, Wang Y, Huang Q, Yuan T, Wang Q, Wang C, Zhou W, Kai G. The AP2/ERF transcription factor SmERF115 positively regulates the biosynthesis of phenolic acids in Salvia miltiorrhiza. J Exp Bot. 2018;70(1): 243-54

8. Ma Y, Yuan L, Wu B, Li X, Chen S, Lu S. Genome-wide identification and characterization of novel genes involved in terpenoid biosynthesis in Salvia miltiorrhiza. J Exp Bot. 2012;63(7):2809-23.

9. Xu Z, Peters RJ, Weirather J, Luo H, Liao B, Zhang X, Zhu Y, Ji A, Zhang B, Hu S, Au K, Song J, Chen S. Full-length transcriptome sequences and splice variants obtained by a combination of sequencing platforms applied to different root tissues of Salvia miltiorrhiza and tanshinone biosynthesis. Plant J. 2015:82(6):951-61.

10. Shani E, Weinstain R, Zhang Y, Castillejo C, Kaiserli E, Chory J, Tsien RY, Estelle M. Gibberellins accumulate in the elongating endodermal cells of Arabidopsis root. Proc Natl Acad Sci U S A. 2012;110(12):4834-9.

11. Yamaguchi S. Gibberellin metabolism and its regulation. Annu Rev Plant Biol. 2008;59(10):225-51.

12. Colebrook EH, Thomas SG, Phillips AL, Hedden P. The role of gibberellin signalling in plant responses to abiotic stress. J Exp Biol. 2014;217(1):67-75.

13. Claeys H, De Bodt SD, Inze D. Gibberellins and DELLAs: central nodes in growth regulatory networks. Trends Plant Sci. 2014;19(4):231-9.

14. Sun T. The molecular mechanism and evolution of the GA-GID1-DELLA signaling module in plants. Curr Biol. 2011;21(9):R338-45.

15. Hakoshima T. Structural basis of the specific interactions of GRAS family proteins. FEBS Lett. 2018;592(4):489-501.

16. Sun $X$, Jones WT, Rikkerink EH. GRAS proteins: the versatile roles of intrinsically disordered proteins in plant signalling. Biochem J. 2012;442(1):1-12.

17. Murase K, Hirano Y, Sun TP, Hakoshima T. Gibberellin-induced DELLA recognition by the gibberellin receptor GID1. Nature. 2008:456(7221):459-63.

18. Yoshida H, Hirano K, Sato T, Mitsuda N, Nomoto M, Maeo K, Koketsu E, Mitani R, Kawamura M, Ishiguro S, Tada Y, Ohme-Takagi M, Matsuoka M, Ueguchi-Tanaka M. DELLA protein functions as a transcriptional activator through the DNA binding of the indeterminate domain family proteins. Proc Natl Acad Sci U S A. 2014;111(9):7861-6.

19. Heo JO, Chang KS, Kim IA, Lee MH, Lee SA, Song SK, Lee MM, Lim J. Funneling of gibberellin signaling by the GRAS transcription regulator SCAR ECROW-LIKE 3 in the Arabidopsis root. Proc Natl Acad Sci U S A. 2011;108(5): 2166-71.

20. Zhang Z, Ogawa M, Fleet CM, Zentella R, Hu J, Heo JO, Lim J, Kamiya Y, Yamaguchi S, Sun T. SCARECROW-LIKE 3 promotes gibberellin signaling by antagonizing master growth repressor DELLA in Arabidopsis. Proc Natl Acad Sci U S A. 2011:108(5):2160-5.

21. Cui $H$, Levesque MP, Vernoux T, Jung JW, Paquette AJ, Gallagher KL, Wang J, Blilou I, Scheres B, Benfey PN. An evolutionarily conserved mechanism delimiting SHR movement defines a single layer of endodermis in plants. Science. 2007:316(5823):421-5.

22. Lucas M, Swarup R, Paponov IA, Swarup K, Casimiro I, Lake D, Peret B, Zappala S, Mairhofer S, Whitworth M, Wang JH, Ljung K, Marchant A, Sandberg G, Holdsworth MJ, Palme K, Pridmore T, Mooney S, Bennett MJ. 
Short-root regulates primary, lateral, and adventitious root development in Arabidopsis. Plant Physiol. 2011;155(1):384-98.

23. Torres-Galea P, Huang L, Chua N, Bolle C. The GRAS protein SCL13 is a positive regulator of phytochrome-dependent red light signaling, but can also modulate phytochrome a responses. Mol Gen Genomics. 2006;276(1): 13-30.

24. Li W, Bai Z, Pei T, Yang D, Mao R, Zhang B, Liu C, Liang Z. SmGRAS1 and SMGRAS2 regulate the biosynthesis of tanshinones and phenolic acids in Salvia miltiorrhiza. Front Plant Sci. 2019;10:1367.

25. Li W, Xing B, Mao R, Bai Z, Yang D, Xu J, Liang Z. SmGRAS3 negatively responds to GA signaling while promotes tanshinones biosynthesis in Salvia miltiorrhiza. Ind Crop Prod. 2020;144:112004.

26. Gong X, Flores-Vergara MA, Hong J, Chu H, Lim J, Franks RG, Liu Z, Xu J. SEUSS integrates gibberellin signaling with transcriptional inputs from the SHR-SCR-SCL3 module to regulate middle cortex formation in the Arabidopsis root. Plant Physiol. 2016;170(3):1675-83.

27. Choe J, Kim B, Yoon EK, Jang S, Kim G, Dhar S, Lee SA, Lim J. Characterization of the GRAS transcription factor SCARECROW-LIKE 28's role in Arabidopsis root growth. JPlant Biol. 2017;60(5):462-71.

28. Ribeiro DM, Araújo WL, Fernie AR, Schippers JHM, Bernd MR. Translatome and metabolome effects triggered by gibberellins during rosette growth in Arabidopsis. J Exp Bot. 2012;63(7):2769-86

29. Fambrini M, Mariotti L, Parlanti S, Salvini M, Pugliesi C. A GRAS-like gene of sunflower (Helianthus annuus L.) alters the gibberellin content and axillary meristem outgrowth in transgenic Arabidopsis plants. Plant Biol. 2015;17(6): 1123-34.

30. Livne S, Lor VS, Nir I, Eliaz N, Aharoni A, Olszewski NE, Eshed Y, Weiss D. Uncovering DELLA-independent gibberellin responses by characterizing new tomato procera mutants. Plant Cell. 2015;27(6):1579-94.

31. Li B, Wang B, Li H, Peng L, Ru M, Liang Z, Yan X, Zhu Y. Establishment of Salvia castanea Diels f. tomentosa Stib. Hairy root cultures and the promotion of tanshinone accumulation and gene expression with $\mathrm{Ag}(+)$, methyl jasmonate, and yeast extract elicitation. Protoplasma. 2016;253(1):1-14.

32. Liu L, Yang DF, Liang T, Zhang H, He Z, Liang Z. Phosphate starvation promoted the accumulation of phenolic acids by inducing the key enzyme genes in Salvia miltiorrhiza hairy roots. Plant Cell Rep. 2016;35(9):1933-42.

33. Mornya $P$, Cheng F. Effect of combined chilling and GA3 treatment on bud abortion in forced 'Luoyanghong' tree peony (Paeonia suffruticosa Andr.). Hortic Plant J. 2018:4(6):250-6.

34. Xu Z, Luo H, Ji A, Zhang X, Song J, Chen L. Global identification of the fulllength transcripts and alternative splicing related to phenolic acid biosynthetic genes in Salvia miltiorrhiza. Front Plant Sci. 2016;7(100):1-10

35. Wang L, Feng Z, Wang X, Wang X, Zhang X. DEGseq: an R package for identifying differentially expressed genes from RNA-seq data. Bioinformatics. 2010;26(1):136-8.

36. Young MD, Wakefield MJ, Smyth GK, Oshlack A. Gene ontology analysis for RNA-seq: accounting for selection bias. Genome Biol. 2010;11(2):R14.

37. Mao X, Cai T, Olyarchuk JG, Wei L. Automated genome annotation and pathway identification using the KEGG Orthology (KO) as a controlled vocabulary. Bioinformatics. 2005;21(19):3787-93.

38. Yang Y, Hou S, Cui G, Chen S, Wei J, Huang L. Characterization of reference genes for quantitative real-time PCR analysis in various tissues of Salvia miltiorrhiza. Mol Biol Rep. 2010;37(1):507-13.

39. Xu W, Chen Z, Ahmed N, Han B, Cui Q, Liu A. Genome-wide identification, evolutionary analysis, and stress responses of the GRAS gene family in Castor beans. Int J Mol Sci. 2016;17(7):E1004.

40. Tamura K, Stecher G, Peterson D, Filipski A, Kumar S. MEGA6: molecular evolutionary genetics analysis version 6.0. Mol Biol Evol. 2013;30(12):2725-9.

\section{Publisher's Note}

Springer Nature remains neutral with regard to jurisdictional claims in published maps and institutional affiliations.

Ready to submit your research? Choose BMC and benefit from:

- fast, convenient online submission

- thorough peer review by experienced researchers in your field

- rapid publication on acceptance

- support for research data, including large and complex data types

- gold Open Access which fosters wider collaboration and increased citations

- maximum visibility for your research: over $100 \mathrm{M}$ website views per year

At BMC, research is always in progress.

Learn more biomedcentral.com/submissions 